\title{
GANGRENE OF THE FUNDUS OF STOMACH
}

Sribatsa Kumar Mohapatra1, Ashok Kumar Nayak², Dhirendra Nath Soren³, Saroj Kumar Panda4, Manoj Kumar Behera ${ }^{5}$

\section{HOW TO CITE THIS ARTICLE:}

Sribatsa Kumar Mohapatra, Ashok Kumar Nayak, Dhirendra Nath Soren, Saroj Kumar Panda, Manoj Kumar Behera. "Gangrene of the Fundus of Stomach". Journal of Evolution of Medical and Dental Sciences 2014; Vol. 3, Issue 49, October 02; Page: 11774-11776, DOI: 10.14260/jemds/2014/3548

INTRODUCTION: The stomach is well known for its rich vascular network which generally protects it from ischemia. So gangrene of the fundus of stomach is a rare event, Its cause has been attributed to gastric volvules, intrathoracic herniation of stomach through the diaphragm, psychogenic polyphagia resulting in massive gastric dilation, ingestion of corrosive materials, embolization of atherosclerotic plague, thrombosis of major arterial supply occlusion of gastric vessels by therapeutically injected foreign bodies and necrotizing gastritis caused by organisms. We report a case of gangrene of funds of stomach that appears to be caused by intake of bhanga. (cannabis sativa)

CASE: A $37 \mathrm{yr}$ old male patient was admitted to the general surgery dept. through emergency dept. with complaints of severe abdominal pain distension of abdomen for one day duration. The pain was severe, sudden, continuous, not associated with nausea or vomiting. No H/O hematemesis or malena. Patient is a known case of tuberculosis $5 \mathrm{yr}$ back and treated with anti-tubercular drugs for $1 \mathrm{yr}$. Patient was addicted to bhang (cannabis sativa). Past medical history was not significant. On examination the patient was afebrile with pulse 96/mm, BP- 110/90 mm Hg. His abdomen was grossly distended with diffuse tenderness, rebound tenderness and absent bowel sound.

Straight x-ray abdomen in erect posture shows dilated bowel loops in right side of abdomen (dilation of small intestine) with no free gas under the diaphragm. Blood sugar, blood urea, serum creatinine, total leucocyte count and electrolytes are within normal limit. We thought a case of intestinal obstruction and planned for exploratory laparotomy. On exploration the peritoneal cavity was filled with hemorrhagic fluid. There was gangrene of fundus of stomach in anterior wall. The small intestine and large intestine were found to be dilated.

The rest of the intra-abdominal organs are found normal. The gangrenous part of the fundus of stomach was resected out and repair of the stomach wall was done. Then feeding jejunostomy was performed. Post-operatively patient was shifted to intensive care unit. Oral fluids were started after clamping the feeding jejunostomy tube on 7 th post-operative day. His general condition gradually improved and liquid diet continued and the patient was discharged from the hospital on $14^{\text {th }}$ day after removing the feeding tube on $10^{\text {th }}$ day.

DISCUSSION: Gangrene of fundus of stomach is a rare and fatal condition. Etiology includes volvulus of stomach, herniation of stomach through the diaphragm, infectious gastritis (Hamad Hisham $2003{ }^{1}$, Richieri et al. $1998^{2}$ ), ingestion of corrosive agents, thromboembolism and occlusion of the major arterial supply, bulimia nervosa, other causes which have been associated with gangrene of fundus of stomach are recent large intake of alcohol, gastritis and upper respiratory tract infections (Madhumita Mukhopadhyay et al ${ }^{3}$.). The Bulimia nervosa (chronic intake of cannabis sativa in large quantity) is the rarest cause of gangrene of fundus of stomach which causes severe gastritis which 
later leads to impaired blood supply to the fundus of stomach and causes gangrene of fundus of stomach. In the present case there was no history suggestive of atherosclerosis neither volvulus nor herniation of stomach as observed intraoperative.

The main vessels were intact ruling out a thromboembolic event. There was no history of ingestion of caustic substance. There were features of peritonitis that might have been caused by transmigration of organisms from the gangrenous fundus of stomach to the peritoneal cavity. Thus the possible cause of gangrene could be due to ingestion of cannabis in large amount as occurs in the psychiatric disorder Bullimia nervosa.

Diagnosis of gangrene of stomach often delayed because of rarity (Malhotra et al $2008{ }^{4}$ ). Initially patients have symptoms of epigastric tenderness, vomiting and may progress to peritonitis and septicemic shock. Radiological tests are nonspecific. Absolute diagnosis is made most frequently at laparotomy (GB Ammori et al $2007^{5}$, Harvey et al $1972{ }^{6}$ ). If a diagnosis of ischemia or gangrene of stomach is considered resuscitation and intravenous antibiotics should be initiated immediately followed by emergency exploratory laparotomy.

Resection of necrotic part of stomach is required with total gastrectomy if necessary. One stage resection with esophago jejunostomy has been reported (Malhotra et al $2008{ }^{4}$ ). Alternative include resection of gangrenous part of fundus and repair of stomach with feeding jejunostomy. A jejunal feeding tube should always be placed (Malhotra et al $2008{ }^{4}$ ) for post-operative feeding. Diagnosis and treatment must be expeditious because mortality rates for gastrectomy due to gangrene of stomach is high.

CONCLUSION: Gangrene of fundus of stomach is a rare disease. The diagnosis is usually made at laparotomy. Treatment consists of resection of gangrenous segment and primary repair. Feeding tube placement in jejunum followed by intravenous antibiotics. Increased awareness of this entity may lead to more prompt diagnosis and increased chance of patient survival. (Malhotra et al., $2008{ }^{4}$ )

\section{REFERENCES:}

1. Hamad Hisham AR. Post vagotomy gastric gangrene due to necrotizing gastritis. The Middle East Journal of Emergency Medicine , 2003 , 3 , 2, 13.

2. Richieri JP, Pol B, Payan MJ. Acute necrotizing ischemic gastritis: clinical, endoscopic and histopathology aspects. Gastrointestinal Endoscopy 1998,48; 210-12.

3. Madhumita Mukhopadhyay, Anil K Saha, Amitava Sarkar, Samarendra N Tripathi, Swapan Biswas, Debasis Mandal, Swadhin Mukherjee. Gastric gangrene due to necrotizing gastritis. Indian Journal of Surgery 73(1)65-67.

4. N Malhorta, S Singla, S Marwah, R Goel, N Marwah, R Taneja. Gangrenous Perforation of Stomach. The Internet Journal of Anesthesiology. 2007 Volume 16 Number 2.

5. Ammori GB, McHugh J and Cimmino VM (2007). Acute gastric necrosis: assessing the risk factors. Surgical Rounds.

6. Harvey RL, Doberneck RC (1972) WC Black. Infarction of the stomach following atheromatous embolization. Gastroenterology 62; 469. 


\section{CASE REPORT}

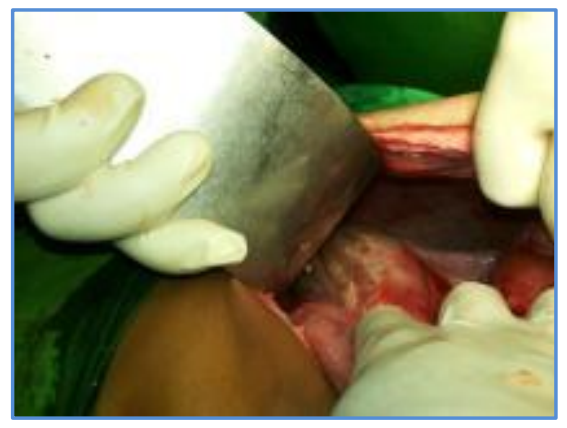

GANGRENOUS PART OF FUNDUS

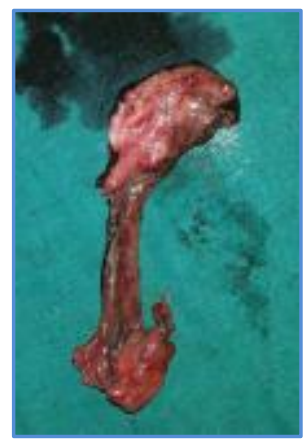

RESECTED GANGRENOUS SEGMENT

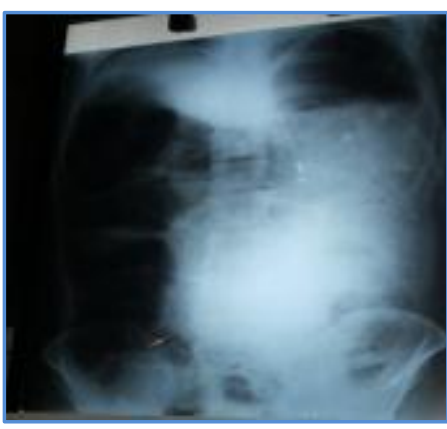

STRAIGHT X RAY OF ABDOMEN

\section{AUTHORS:}

1. Sribatsa Kumar Mohapatra

2. Ashok Kumar Nayak

3. Dhirendra Nath Soren

4. Saroj Kumar Panda

5. Manoj Kumar Behera

\section{PARTICULARS OF CONTRIBUTORS:}

1. Professor \& HOD, Department of General Surgery, Veer Surendra Sai Medical College, Burla, Sambalpur.

2. Assistant Professor, Department of General Surgery, Veer Surendra Sai Medical College, Burla, Sambalpur.

3. Assistant Professor, Department of General Surgery, Veer Surendra Sai Medical College, Burla, Sambalpur.
4. Post Graduate Student, Department of General Surgery, Veer Surendra Sai Medical College, Burla, Sambalpur.

5. Post Graduate Student, Department of General Surgery, Veer Surendra Sai Medical College, Burla, Sambalpur

\section{NAME ADDRESS EMAIL ID OF THE CORRESPONDING AUTHOR:}

Dr. Saroj Kumar Panda,

PG Hostel, Room No. 6,

VSS Medical College,

Burla, Sambalpur,Odisha.

Email: sarojvss38@gmail.com

Date of Submission: 08/09/2014.

Date of Peer Review: 09/09/2014.

Date of Acceptance: 17/09/2014.

Date of Publishing: 01/10/2014. 